\title{
Nurse Led Follow Up: Is It The Best Way Forward for Post- Operative Endometriosis Patients?
}

\author{
R Mallick ${ }^{*}$, Z Magama, C Neophytou, R Oliver, F Odejinmi \\ Barts Health NHS Trust, Whipps Cross University Hospital, London, UK
}

*Corresponding Author: R Mallick, Barts Health NHS Trust, Whipps Cross University Hospital, London, UK; E-mail: rmallick@doctors.org.uk

Received: 03 April 2018; Accepted: 09 April 2018; Published: 12 April 2018

\begin{abstract}
The benefits of nurse led follow-up are well documented in the wider literature and include higher patient satisfaction rates, improved information delivery and quicker discharge rates. Unfortunately there is a paucity of data when it comes to benign gynaecology and the aim of this study was to establish the efficacy of outpatient services offered by an endometriosis specialist nurse compared to standard medical follow up in women undergoing operative laparoscopy for severe endometriosis. Outcome measures included both patient satisfaction and the duration of follow-up until discharge. Our findings highlight that a nurse led service is highly acceptable to patients and leads not only to higher satisfaction rates, but also a reduced duration of follow-up resulting in a significantly more cost effective service without any detriment to the patient.
\end{abstract}

Keywords: Endometriosis; Nurse

\section{Introduction}

Endometriosis remains a challenging condition to manage and ideally patients with severe endometriosis should be managed in a tertiary referral BSGE (British Society for Gynaecological Endoscopy) centre where they will have access to a more holistic and multi-disciplinary approach to their care including contact with an endometriosis clinical specialist nurse (CNS).

The benefits of a nurse led service are numerous and widely documented in the literature, particularly in the medical setting [1-3]. However when it comes to benign gynaecological conditions and specifically endometriosis, there is a 
paucity of data. The role of the endometriosis nurse is not only invaluable in the early stages of diagnosis and management, particularly with regards to patient support and counselling [4, 5], but they also play a fundamental role in the post-operative period supporting the patient, offering them a readily available port of call and taking them through the treatment pathway to a successful discharge. In this day in age of non-team based medical training, it is very rare for the patient to be seen by the same junior medical member of staff and thus the continuity of care a endometriosis nurse offers is invaluable.

The aim of this study was to establish the efficacy of outpatient services offered by an endometriosis CNS, compared to standard medical follow up, in women undergoing operative laparoscopy for severe endometriosis assessing both patient satisfaction and the duration of follow-up until discharge.

\section{Materials and methods}

A prospective cohort study was undertaken on women undergoing operative laparoscopy for severe endometriosis and receiving outpatient follow-up led by either a CNS or a gynaecology specialist registrar. The primary outcome was patient satisfaction levels, with the secondary outcome being the duration of follow-up until discharge from outpatient endometriosis services. The inclusion criteria were duration of symptoms for over 6 months prior to surgery and the presence of severe endometriosis (stage 3 or 4) diagnosed during laparoscopy.

Patients were asked to complete a Likert satisfaction scale 3 months post-operatively, after having received regular outpatient clinic follow-up. The BSGE pelvic pain questionnaire was used to collect information on the severity of symptoms, previous treatment and quality of life before operative laparoscopy, as well as 6 months and 1 year postoperatively. The duration of outpatient clinic follow-up, from the time of operation until complete discharge from clinic, was also recorded.

Data is presented as the mean+/-standard error of the mean (SEM). All data was normally distributed and comparison between the 2 groups was undertaken using the unpaired t-test. The threshold for statistical significance was set at $\mathrm{p}<0.05$. The data was analysed using Prism software (version 7). Ethical approval was sought but not required, as this was a review of clinical services.

\section{Results}

A total of 74 women were included in this cohort study. 50 women received outpatient clinic follow-up by the CNS and twenty-four by the gynaecology registrar. Baseline characteristics were comparable between the two groups. No statistical difference was detected between the two groups in the reporting of symptoms, severity of disease or quality of life before operative laparoscopy or at 6 months and 1 year post-operatively. More than 95\% of patients in each group underwent a one-stage procedure with recto-vaginal resection. The rates of post-operative complications were comparable between the two groups. 
The mean overall satisfaction rate as reported by patients on a Likert satisfaction scale was $8.0 \pm 0.2$ and $5.3 \pm 0.5$ out of a maximum of 10 for the CNS and registrar groups respectively $(\mathrm{p}<0.05)$ (Figure 1). The average duration of follow-up from the time of operation to complete discharge from outpatient endometriosis services was $29.0 \pm 3.3$ and $44.8 \pm 7.8$ weeks for the CNS and registrar groups respectively $(\mathrm{p}<0.05)$, accounting for a 1.5 times earlier discharge for patients followed-up by the CNS (Figure 2).

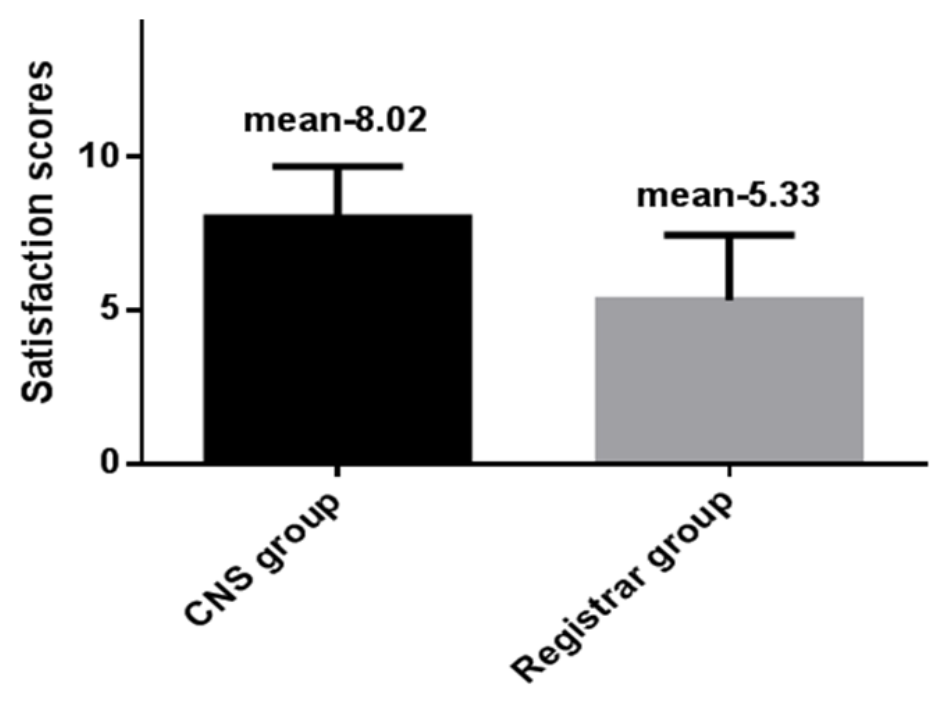

Figure 1: Overall satisfaction scores

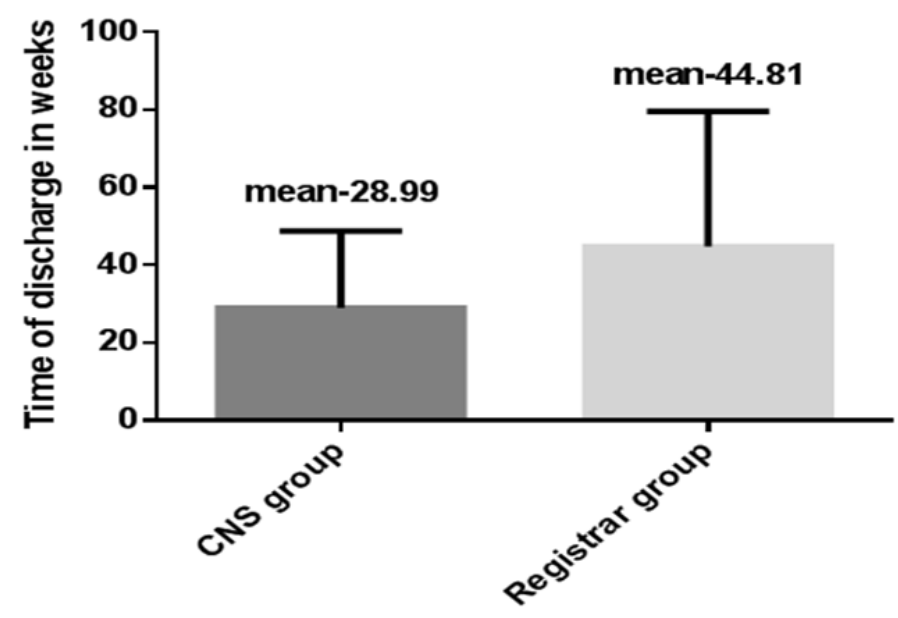

Figure 2: Duration of follow-up from operation to discharge

\section{Discussion}

Our findings highlights that follow up of patients with severe endometriosis by a CNS is not only acceptable to patients, but patients tend to prefer this with higher satisfaction rates recorded when compared to standard follow up. 
Although data in an endometriosis setting is limited, these high satisfaction rates are well documented in the wider literature from a multitude of medical settings [2, 4, 6]. Furthermore a randomized controlled trial by Dawes et al. which compared specialist nurse supported discharge with routine hospital care in a benign gynaecological setting found a significantly reduced post-operative length of hospital stay, improved information delivery and patient satisfaction in the specialist nurse group [7].

The patients undergoing nurse led follow-up were also discharged quicker, making the service more cost effective. Although carried out in a different setting this compares favourably with the findings of Graham et al, who found that following day case laparoscopic surgery patients in the nurse led discharge group were much likely to be discharged quicker than in the doctor led group [8].

\section{Conclusion}

Endometriosis is a challenging condition to manage and patients benefit significantly from a holistic multidisciplinary approach with early input and support from an endometriosis CNS. Post-operatively developing a service allowing these patients to be followed up by the CNS facilitates an extension of the continuity of care and results in higher satisfaction rates and reduced duration of follow-up resulting in a significantly more cost effective service without any detriment to the patient.

\section{Declaration of interest}

The authors declare that there is no conflict of interest regarding the publication of this article.

\section{References}

1. Moore S, Corner J, Haviland J, Wells M, Salmon E, Normand C, et al. Nurse led follow up and conventional medical follow up in management of patients with lung cancer: randomised trial. BMJ 325 (2002): 1145.

2. Lewis R, Neal RD, Williams NH, France B, Wilkinson C, Hendry M, et al. Nurse-led vs. conventional physician-led follow-up for patients with cancer: systematic review. J Adv Nurs 65 (2009): 706-723.

3. Laurant M, Reeves D, Hermens R, Braspenning J, Grol R, Sibbald B. Substitution of doctors by nurses in primary care. Cochrane Database Syst Rev 2005 (2): Cd001271.

4. Courtenay M, Carey N. The impact and effectiveness of nurse-led care in the management of acute and chronic pain: a review of the literature. J Clin Nurs 17 (2018): 2001-2013.

5. Cambitzi J, Nagaratnam M. Endometriosis-associated pain syndrome: a nurse-led approach. British Journal of Pain 7 (2013): 31-38.

6. Hill J. Patient satisfaction in a nurse-led rheumatology clinic. Journal of Advanced Nursing 25 (1997): 347-354.

7. Dawes HA, Docherty T, Traynor I, Gilmore DH, Jardine AG, Knill-Jones R. Specialist nurse supported discharge in gynaecology: A randomised comparison and economic evaluation. European Journal of 
Obstet Gynecol Res 2018; 1 (2): 028-032

DOI: 10.26502/ogr005

Obstetrics \& Gynecology and Reproductive Biology 130 (2007): 262-270.

8. Graham L, Neal CP, Garcea G, Lloyd DM, Robertson GS, Sutton CD. Evaluation of nurse-led discharge following laparoscopic surgery. Journal of Evaluation in Clinical Practice 8 (2012): 19-24.

Citation: R Mallick, Z Magama, C Neophytou, R Oliver, F Odejinmi. Nurse Led Follow Up: Is It The Best Way Forward for Post-Operative Endometriosis Patients?. Obstetrics and Gynecology Research 1 (2018): 028-032.

(C) This article is an open access article distributed under the terms and conditions of the Creative Commons Attribution (CC-BY) license 4.0 